\title{
Optimal convergence rates for semidiscrete finite element approximations of linear space-fractional partial differential equations under minimal regularity assumptions
}

\author{
Fang Liu ${ }^{\mathrm{a}}$, Zongqi Liang ${ }^{\mathrm{b}}$, Yubin Yan ${ }^{*, c}$ \\ ${ }^{a}$ Department of Mathematics, Luliang University, P. R. China \\ ${ }^{b}$ School of Sciences, Jimei University, Xiamen 361021, P. R. China \\ ${ }^{c}$ Department of Mathematics, University of Chester, CH1 4BJ, UK
}

\begin{abstract}
We consider the optimal convergence rates of the semidiscrete finite element approximations for solving linear space-fractional partial differential equations by using the regularity results for the fractional elliptic problems obtained recently by Jin et al. [20] and Ervin et al. [9]. The error estimates are proved by using two approaches. One approach is to apply the duality argument in Johnson [22] for the heat equation to consider the error estimates for the linear space-fractional partial differential equations. This argument allows us to obtain the optimal convergence rates under the minimal regularity assumptions for the solution. Another approach is to use the approximate solution operators of the corresponding fractional elliptic problems. This argument can be extended to consider more general linear space-fractional partial differential equations. Numerical examples are given to show that the numerical results are consistent with the theoretical results.
\end{abstract}

Key words:

space-fractional partial differential equations; finite element method; error estimates.

AMS Subject Classification: 65M12; 65M06; 65M70;35S10

\section{Introduction}

In this paper we will consider a finite element method for solving the following linear space-fractional partial differential equation, with $1 / 2<\alpha<1$, [2], [48]

$$
\begin{aligned}
& \frac{\partial u(x, t)}{\partial t}-{ }_{0}^{R} D_{x}^{2 \alpha} u(x, t)=f(x, t), \quad 0<t<T, 0<x<1, \\
& u(0, t)=u(1, t)=0, \quad 0<t<T, \\
& u(x, 0)=u_{0}(x), \quad 0<x<1,
\end{aligned}
$$

or $[2],[48]$

$$
\begin{aligned}
& \frac{\partial u(x, t)}{\partial t}+\mathcal{L}^{2 \alpha} u(x, t)=f(x, t), \quad 0<t<T, 0<x<1, \\
& u(0, t)=u(1, t)=0, \quad 0<t<T \\
& u(x, 0)=u_{0}(x), \quad 0<x<1
\end{aligned}
$$

\footnotetext{
${ }^{*}$ Corresponding author [copyright reserved]

Email addresses: 13935870281@163.com (Fang Liu), zqliang@jmu.edu.cn (Zongqi Liang), y.yan@chester.ac.uk (Yubin Yan )

Preprint submitted to Elsevier

December 13, 2018
} 
where the two-sided fractional derivative operator $\mathcal{L}^{2 \alpha}$ is defined by [33], [35]

$$
\mathcal{L}^{2 \alpha} w(x)=-\frac{1}{2}\left({ }_{0}^{R} D_{x}^{2 \alpha} w(x)+{ }_{x}^{R} D_{1}^{2 \alpha} w(x)\right) .
$$

Here ${ }_{0}^{R} D_{x}^{\gamma} w(x)$ and ${ }_{x}^{R} D_{1}^{\gamma} w(x), 1<\gamma<2$ are called the left-sided and right-sided Riemann-Liouville fractional derivatives defined by

$$
{ }_{0}^{R} D_{x}^{\gamma} w(x)=\frac{1}{\Gamma(2-\gamma)} \frac{d^{2}}{d x^{2}} \int_{0}^{x}(x-y)^{1-\gamma} w(y) d y, \quad{ }_{x}^{R} D_{1}^{\gamma} w(x)=\frac{1}{\Gamma(2-\gamma)} \frac{d^{2}}{d x^{2}} \int_{x}^{1}(y-x)^{1-\gamma} w(y) d y .
$$

Space-fractional partial differential equations are widely used to model complex phenomena, such as system biology [41], physics [30], [45], chemistry and biochemistry [40], finance [32], hydrology [1], thermodynamics [36], [44], quasi-geostrophic flow [5], etc.

In recent years, many authors consider the numerical methods for solving space-fractional partial differential equations. Meerschaert et al. [28] [29] [37] considered the finite difference methods for solving fractional advection-dispersion equations and diffusion equations. Yang et al. [39] considered the finite difference methods for solving two-sided fractional partial differential equations. Ford et al. [14] studied the finite difference methods for two-sided space-fractional partial differential equations. Ervin and Roop [10] introduced the variational formulation for solving space-fractional advection dispersion equation by using finite element methods, see also [13]. Burrage et al. [4] considered finite element methods for solving space-fractional reaction-diffusion equation. Deng and Hesthaven [7] studied the discontinuous Galerkin methods for fractional diffusion equation. Zhang et al. [48] studied the finite element methods for solving symmetric space-fractional partial differential equations, see also [2], [26]. Li and $\mathrm{Xu}$ [23] [24] considered the spectral methods for solving linear space-fractional partial differential equations. Bueno-Orovio et al. [3] studied the Fourier spectral methods for solving space-fractional reaction-diffusion equation. Zeng et al. [46] considered the spectral methods for two-sided space-fractional partial differential equations. Ilic et al. [18] [19] introduced the so-called matrix transfer technique (MTT) for solving space-fractional partial differential equation. Qin et al. [34] considered a two-dimensional multi-term time and space fractional Bloch-Torry model based on the rectangular finite elements. Feng et al. [12] considered the finite element methods for solving time-space Riesz fractional diffusion equation on irregular convex domain in two dimensional case. A novel unstructured mesh finite element method for solving time-space fractional wave equation in irregular convex domain in two dimensional case was studied in Fan et al. [11]. Zhao et al. [42], [43] studied the nonconforming finite element methods for solving time fractional diffusion equations.

See [47], [6], [17], [27], [21], [16], [31], [25], [15], [8], etc. for further references of the numerical methods for solving time-space fractional partial differential equations.

To consider the error estimates of the finite element methods for solving space-fractional partial differential equations, one has to use the regularity results for the solution of the corresponding fractional elliptic problem. Many available error estimates in literature for the finite element methods for solving space-fractional partial differential equations were proved based on the assumptions (without proof) on the regularity of the solution of the corresponding fractional elliptic problem. Recently Jin et al. [20] discussed the regularity of the solution of the following fractional elliptic problem, with $1 / 2<\alpha<1, g \in L^{2}(0,1)$,

$$
\begin{aligned}
& -{ }_{0}^{R} D_{x}^{2 \alpha} w(x)=g(x), 0<x<1, \\
& w(0)=w(1)=0,
\end{aligned}
$$

and showed that $w \in \tilde{H}_{L}^{2 \alpha-1+\beta}, 0<\beta<1 / 2$ for $g \in L^{2}(0,1)$, where the space $\tilde{H}_{L}^{2 \alpha-1+\beta}$ is defined in Jin et al. [20]. Ervin et al. [9] considered the following two-sided fractional elliptic problem, with $1 / 2<\alpha<1$, $g \in L^{2}(0,1)$,

$$
\begin{aligned}
& \mathcal{L}^{2 \alpha} w(x)=g(x), 0<x<1, \\
& w(0)=w(1)=0
\end{aligned}
$$


and showed that $w \in H^{\alpha+\beta}(0,1), 0<\beta<1 / 2$ for $g \in L^{2}(0,1)$, where the space $H^{\alpha+\beta}(0,1)$ denotes the standard Sobolev space.

In this paper we will consider the error estimates of the semidiscrete finite element approximation for solving (1)-(3) and (4)-(6), respectively by using the regularity results obtained recently in Jin et al. [20] and Ervin et al. [9]. Our error estimates are based on the argument developed in Johnson [22] for the finite element analysis of the heat equation. This argument allows us to obtain the optimal convergence rates under the minimal regularity assumption for the solution $u$. More precisely we only require $u \in L^{\infty}\left(0, T ; H^{r}(0,1)\right)$ for some $r>0$ and there is no derivative $\frac{\partial u}{\partial t}$ involved in the error bound. Recall that most error estimates of the finite element methods for solving (1)-(3) and (4)-(6) in literature require the regularity of the derivative $\frac{\partial u}{\partial t}$ in the error bound explicitly, for example, $\frac{\partial u}{\partial t} \in L^{1}\left(0, T ; H^{r}(0,1)\right)$ for some $r>0$. We will also consider in this paper the error estimates of the semidiscrete finite element approximation for solving (1)-(3) and (4)-(6) by using the approximate solution operators of the corresponding fractional elliptic problems. This argument can be extended to consider more general space-fractional partial differential equations.

The main contributions of this paper are the following:

- Obtain the optimal convergence rates of the semidiscrete finite element approximations for solving linear space-fractional partial differential equations by using the regularity results obtained recently by Jin et al. [20] and Ervin et al. [9]. Such error estimates in literature are often proved based on the assumptions (without proof) on the regularity of the solutions.

- Obtain the optimal convergence rates of the semidiscrete finite element approximations for solving linear space-fractional partial differential equations under the minimal regularity requirements for the solution $u$, i.e., $u \in L^{\infty}\left(0, T ; H^{r}(0,1)\right)$ for some $r>0$ by using the duality argument in Johnson [22]. We have not seen any work in literature which applied the duality argument in Johnson [22] to consider the error estimates of the semidiscrete finite element approximations for solving linear space-fractional partial differential equations.

- Prove the error estimates of the semidiscrete finite element approximations for solving linear spacefractional partial differential equations by using the approximate solution operators. This argument can be extended to consider more general space-fractional partial differential equations.

The paper is organized as follows. In Section 2, we consider the finite element formulation of the spacefractional partial differential equation. In Section 3, we prove the error estimates by using a duality argument. In Section 4, we prove the error estimates by using the approximate solution operator $T_{h, \alpha}, 1 / 2<\alpha<1$. Finally in Section 5, we give some numerical results.

By $C$ we denote a positive constant independent of the functions and parameters concerned, but not necessarily the same at different occurrences.

\section{Finite element method}

In this section, we will consider finite element method for solving (1)-(3) or (4)-(6). We first introduce some fractional Sobolev spaces.

Definition 2.1. [10], [23] For any $\sigma>0$, we define the spaces ${ }^{l} H_{0}^{\sigma}(0,1)$ and ${ }^{r} H_{0}^{\sigma}(0,1)$ to be the closures of $C_{0}^{\infty}(0,1)$ with respect to the norms $\|v\|_{{ } H_{0}^{\sigma}(0,1)}$ and $\|v\|_{{ }^{r} H_{0}^{\sigma}(0,1)}$, respectively, where

$$
\|v\|_{{ }^{H} H_{0}(0,1)}^{2}:=\|v\|_{L^{2}(0,1)}^{2}+\left\|{ }_{0}^{R} D_{x}^{\sigma} v\right\|_{L^{2}(0,1)}^{2},
$$

and

$$
\|v\|_{{ }^{H} H_{0}^{\sigma}(0,1)}^{2}:=\|v\|_{L^{2}(0,1)}^{2}+\left\|_{x}^{R} D_{1}^{\sigma} v\right\|_{L^{2}(0,1)}^{2} .
$$

The semi-norms are defined by $|v|_{{ } H_{0}^{\sigma}(0,1)}:=\left\|{ }_{0}^{R} D_{x}^{\sigma} v\right\|_{L^{2}(0,1)}$ and $|v|_{{ }^{r} H_{0}^{\sigma}(0,1)}:=\left\|{ }_{x}^{R} D_{1}^{\sigma} v\right\|_{L^{2}(0,1)}$, respectively. 
Definition 2.2. [10], [23] For any $\sigma>0, \sigma \neq n-1 / 2, n \in \mathbb{Z}^{+}$, we define the space ${ }^{c} H_{0}^{\sigma}(0,1)$ to be the closure of $C_{0}^{\infty}(0,1)$ with respect to the norm $\|v\|^{c} H_{0}^{\sigma}(0,1)$, where

$$
\|v\|_{{ }^{\prime} H_{0}^{\sigma}(0,1)}^{2}:=\|v\|_{L^{2}(0,1)}^{2}+\left|\left({ }_{0}^{R} D_{x}^{\sigma} v,{ }_{x}^{R} D_{1}^{\sigma} v\right)\right| .
$$

The semi-norm is defined by $|v|_{c}^{2} H_{0}^{\sigma}(0,1):=\left|\left({ }_{0}^{R} D_{x}^{\sigma} v,{ }_{x}^{R} D_{1}^{\sigma} v\right)\right|$.

Definition 2.3. [10], [23] For any $\sigma>0$, let $H^{\sigma}(\mathbb{R}), \sigma>0$ denote the fractional Sobolev space defined in the whole line $\mathbb{R}$. We define

$$
H^{\sigma}(0,1)=\left\{v \in L^{2}(0,1):\left.\tilde{v}\right|_{(0,1)}=v, \text { where } \tilde{v} \in H^{\sigma}(\mathbb{R})\right\}
$$

with the norm

$$
\|v\|_{H^{\sigma}(0,1)}=\inf _{\tilde{v} \in H^{\sigma}(\mathbb{R}),\left.\tilde{v}\right|_{(0,1)=v}}\|\tilde{v}\|_{H^{\sigma}(\mathbb{R})},
$$

where $\|\tilde{v}\|_{H^{\sigma}(\mathbb{R})}=\left\|\left(1+|w|^{2}\right)^{\sigma / 2} \mathcal{F}(\tilde{v})(w)\right\|_{L^{2}(\mathbb{R})}$, and $\mathcal{F}(\tilde{v})$ denotes the Fourier transform of $\tilde{v}$ and the corresponding semi-norm is defined by $|\tilde{v}|_{H^{\sigma}(\mathbb{R})}=\left\||w|^{\sigma} \mathcal{F}(\tilde{v})\right\|_{L^{2}(\mathbb{R})}$. Further we define the Sobolev space $H_{0}^{\sigma}(0,1)$ to be the closure of $C_{0}^{\infty}(0,1)$ with respect to the norm $\|v\|_{H^{\sigma}(0,1)}$. The semi-norm in $H_{0}^{\sigma}(0,1)$ is denoted by $|\cdot|_{H_{0}^{\sigma}(0,1)} \cdot$

Lemma 2.1. [10], [23] Let $\sigma>0, \sigma \neq n-1 / 2, n \in \mathbb{Z}^{+}$. The semi-norms in spaces ${ }^{l} H_{0}^{\sigma}(0,1),{ }^{r} H_{0}^{\sigma}(0,1),{ }^{c} H_{0}^{\sigma}(0,1)$ and $H_{0}^{\sigma}(0,1)$ are equivalent and the norms in spaces ${ }^{l} H_{0}^{\sigma}(0,1),{ }^{r} H_{0}^{\sigma}(0,1),{ }^{c} H_{0}^{\sigma}(0,1)$ and $H_{0}^{\sigma}(0,1)$ are also equivalent.

Below we will denote $(\cdot, \cdot)$ and $\|\cdot\|$ as the inner product and norm in $L^{2}(0,1)$, respectively.

Lemma 2.2. [10], [23] Let $\sigma>0, \sigma \neq n-1 / 2, n \in \mathbb{Z}^{+}$, we have

$$
\left({ }_{0}^{R} D_{x}^{\sigma} v,{ }_{x}^{R} D_{1}^{\sigma} v\right)=\cos (\pi \sigma)\left\|_{0}^{R} D_{x}^{\sigma} v\right\|^{2}, \forall v \in H_{0}^{\sigma}(0,1) .
$$

In particular, $\left({ }_{0}^{R} D_{x}^{\sigma} v,{ }_{x}^{R} D_{1}^{\sigma}\right)$ is negative when $1 / 2<\sigma \leq 1$.

Proof: It is sufficient to prove

$$
\left({ }_{0}^{R} D_{x}^{\sigma} \varphi,{ }_{x}^{R} D_{1}^{\sigma} \varphi\right)=\cos (\pi \sigma)\left\|_{0}^{R} D_{x}^{\sigma} \varphi\right\|^{2}, \forall \varphi \in C_{0}^{\infty}(0,1) .
$$

In fact, we have, for any $\varphi \in C_{0}^{\infty}(0,1),[23]$,

$$
\left({ }_{0}^{R} D_{x}^{\sigma} \varphi,{ }_{x}^{R} D_{1}^{\sigma} \varphi\right)=\left({ }_{-\infty}^{R} D_{x}^{\sigma} \tilde{\varphi},{ }_{x}^{R} D_{\infty}^{\sigma} \tilde{\varphi}\right)_{L^{2}(\mathbb{R})}=\cos (\pi \sigma)\left\|_{-\infty}^{R} D_{x}^{\sigma} \tilde{\varphi}\right\|_{L^{2}(\mathbb{R})}^{2}=\cos (\pi \sigma)\left\|_{0}^{R} D_{x}^{\sigma} \varphi\right\|^{2},
$$

where $\tilde{\varphi}$ is the extension of $\varphi$ by zero outside of $(0,1)$.

Lemma 2.3. [10], [23] Let $1 / 2<\alpha<1$. We have, see [23],

$$
\begin{aligned}
& \left({ }_{0}^{R} D_{x}^{2 \alpha} w, v\right)=\left({ }_{0}^{R} D_{x}^{\alpha} w,{ }_{x}^{R} D_{1}^{\alpha} v\right), \forall w, v \in H_{0}^{\alpha}(0,1), \\
& \left({ }_{x}^{R} D_{1}^{2 \alpha} w, v\right)=\left({ }_{x}^{R} D_{1}^{\alpha} w,{ }_{0}^{R} D_{x}^{\alpha} v\right), \forall w, v \in H_{0}^{\alpha}(0,1) .
\end{aligned}
$$

We also have the following fractional Poincaré inequality, see [10], [13], [23],

Lemma 2.4. For $u \in H_{0}^{\alpha}(0,1), 1 / 2<\alpha<1$, we have

$$
\|u\|_{L^{2}(0,1)} \leq C|u|_{H_{0}^{\alpha}(0,1)}
$$

and for $0<s<\mu, s \neq n-1 / 2, n \in \mathbb{Z}^{+}$,

$$
|u|_{H_{0}^{s}(0,1)} \leq C|u|_{H_{0}^{\mu}(0,1)} .
$$


Multiplying $v \in H_{0}^{\alpha}(0,1), 1 / 2<\alpha<1$ in both sides of the equation (1) or (4) and integrating on $(0,1)$ we obtain the following variational form for (1)-(3) or (4)-(6): find $u \in H_{0}^{\alpha}(0,1)$ such that, by Lemma 2.3 ,

$$
\begin{aligned}
& \left(u_{t}, v\right)+B_{\alpha}(u, v)=(f, v), \quad \forall v \in H_{0}^{\alpha}(0,1), \\
& u(0)=u_{0}
\end{aligned}
$$

where the bilinear form $B_{\alpha}(\cdot, \cdot)$ is defined by

$$
B_{\alpha}(u, v)=-\left({ }_{0}^{R} D_{x}^{\alpha} u,{ }_{x}^{R} D_{1}^{\alpha} v\right),
$$

or

$$
B_{\alpha}(u, v)=\frac{1}{2}\left(\left({ }_{0}^{R} D_{x}^{\alpha} u,{ }_{x}^{R} D_{1}^{\alpha} v\right)+\left({ }_{x}^{R} D_{1}^{\alpha} u,{ }_{0}^{R} D_{x}^{\alpha} v\right)\right)
$$

respectively.

Let $S_{h} \subset H_{0}^{\alpha}(0,1), 1 / 2<\alpha<1$ be the continuous piecewise linear finite element space. The finite element method of (12)-(13) is to find $u_{h}(t) \in S_{h}$ such that

$$
\begin{aligned}
& \left(u_{h, t}, \chi\right)+B_{\alpha}\left(u_{h}, \chi\right)=(f, \chi), \quad \forall \chi \in S_{h}, \\
& u_{h}(0)=u_{0 h},
\end{aligned}
$$

where $u_{0 h} \in S_{h}$ is some appropriate approximation of $u_{0} \in L^{2}(0,1)$ in $S_{h}$.

\section{Error estimates proved by using a duality argument}

\subsection{The left-sided Riemann-Liouville fractional derivative case}

In this subsection, we will consider the error estimates of the finite element methods for solving (12)(13) in the left-sided Riemann-Liouville fractional derivative case. To do this, we first need to consider the fractional elliptic problem (8)-(9). The variational form of (8)-(9) is to find $w \in H_{0}^{\alpha}(0,1)$, such that

$$
B_{\alpha}(w, \psi)=(g, \psi), \quad \forall \psi \in H_{0}^{\alpha}(0,1),
$$

where $B_{\alpha}(\cdot, \cdot)$ is defined by $(14)$. Since $B_{\alpha}(\cdot, \cdot)$ is continuous and coercive on $H_{0}^{\alpha}(0,1), 1 / 2<\alpha<1$, see Jin et al. [20, Lemma 4.2], by Riesz representation theorem, there exists a unique bounded linear operator $\tilde{A}_{\alpha}: H_{0}^{\alpha}(0,1) \rightarrow\left(H_{0}^{\alpha}(0,1)\right)^{\prime}$, such that

$$
B_{\alpha}(\varphi, \psi)=\left\langle\tilde{A}_{\alpha} \varphi, \psi\right\rangle, \quad \forall \varphi, \psi \in H_{0}^{\alpha}(0,1) .
$$

Here $\left(H_{0}^{\alpha}(0,1)\right)^{\prime}$ denotes the dual space of $H_{0}^{\alpha}(0,1)$ and $\langle\cdot, \cdot\rangle$ denotes the duality pairing between $H_{0}^{\alpha}(0,1)$ and $\left(H_{0}^{\alpha}(0,1)\right)^{\prime}$.

Define

$$
\mathcal{D}\left(A_{\alpha}\right)=\left\{\psi \in H_{0}^{\alpha}(0,1): \tilde{A}_{\alpha} \psi \in L^{2}(0,1)\right\}
$$

and an operator $A_{\alpha}: \mathcal{D}\left(A_{\alpha}\right) \rightarrow L^{2}(0,1)$ by

$$
B_{\alpha}(\varphi, \psi)=\left(A_{\alpha} \varphi, \psi\right), \quad \forall \varphi \in \mathcal{D}\left(A_{\alpha}\right), \psi \in H_{0}^{\alpha}(0,1) .
$$

Then we may write (8)-(9) as the following abstract form, with $g \in L^{2}(0,1)$,

$$
A_{\alpha} w=g
$$

The domain $\mathcal{D}\left(A_{\alpha}\right)$ has a complicated structure. For example, Jin et al. [21, Remark 2.1] showed that the corresponding domain $\mathcal{D}\left(A_{\alpha}\right)$ of $A_{\alpha}$ consists of functions of the form $I_{0}^{2 \alpha} g-\left(I_{0}^{2 \alpha} g\right) x^{2 \alpha-1}$ where $g \in L^{2}(0,1)$ 
and $x^{2 \alpha-1} \in \tilde{H}_{L}^{2 \alpha-1+\beta}(0,1)$ for any $0 \leq \beta<1 / 2$. Hence $\mathcal{D}\left(A_{\alpha}\right) \subset \tilde{H}_{L}^{2 \alpha-1+\beta}(0,1) \cap H_{0}^{\alpha}(0,1)$ which implies that the solution $w$ of (8)-(9) satisfies

$$
\|w\|_{\tilde{H}_{L}^{2 \alpha-1+\beta}(0,1)} \leq\left\|A_{\alpha} w\right\|_{L^{2}(0,1)}=\|g\|_{L^{2}(0,1)} .
$$

Here $I_{0}^{2 \alpha}$ denotes the Riemann-Liouville fractional integral operator and $\tilde{H}_{L}^{2 \alpha-1+\beta}(0,1)$ is defined in Jin et al. [20], [21].

Remark 3.1. The parameter $\beta \in[0,1 / 2)$ is introduced to describe the regularity $w$ in (19) for any $g \in$ $L^{2}(0,1)$. For $\alpha \in(1 / 2,1]$ and $g \in L^{2}(0,1)$, the best regularity satisfied for $w$ in (19) is $w \in \tilde{H}^{r}$ where $r \approx 2 \alpha-1+\beta$ with $\beta=1 / 2$, that is $r \approx 2 \alpha-1 / 2$, but $r \neq 2 \alpha-1 / 2$. In Examples 5.1-5.3 in Section 5 , we compare the experimentally determined orders of convergence "EOC "with the best theoretical orders $2(r-\alpha)=2((2 \alpha-1+\beta)-\alpha) \approx 2 \alpha-1$ obtained by choosing $\beta=1 / 2$.

We now introduce the fractional Ritz projection $R_{h, \alpha}$ on $S_{h}$.

Definition 3.1. Let $1 / 2<\alpha<1$ and let $v \in H_{0}^{\alpha}(0,1)$. We define $R_{h, \alpha}: H_{0}^{\alpha}(0,1) \rightarrow S_{h}$ by

$$
B_{\alpha}\left(R_{h, \alpha} v, \chi\right)=B_{\alpha}(v, \chi), \quad \forall \chi \in S_{h}, \quad v \in H_{0}^{\alpha}(0,1) .
$$

It is easy to see that $R_{h, \alpha}: H_{0}^{\alpha}(0,1) \rightarrow S_{h}$ is well defined since $B_{\alpha}(\cdot, \cdot)$ is continuous and coercive on $S_{h}$, see Jin et al. [20, Lemma 5.2]. Further we have

Lemma 3.1. Let $v \in H^{r}(0,1) \cap H_{0}^{\alpha}(0,1), r=2 \alpha-1+\beta, 1-\alpha \leq \beta<1 / 2,1 / 2<\alpha<1$ and let $R_{h, \alpha}$ : $H_{0}^{\alpha}(0,1) \rightarrow S_{h}$ be the fractional Ritz projection onto $S_{h}$ defined as in (21). Then, there exists a constant $C=C(\alpha, \beta)$ such that

$$
\left\|R_{h, \alpha} v-v\right\|+h^{r-\alpha}\left\|R_{h, \alpha} v-v\right\|_{H_{0}^{\alpha}(0,1)} \leq C h^{2(r-\alpha)}\|v\|_{H^{r}(0,1)} .
$$

Here and below $\|\cdot\|_{H^{r}(0,1)}$ denotes the standard Sobolev norm in $H^{r}(0,1)$. $4.2]$,

To prove this lemma, we need the following interpolation error estimates. We have, see [10, Theorem

Lemma 3.2. Let $v \in H^{s}(0,1) \cap H_{0}^{\alpha}(0,1), \alpha \leq s \leq 2 \alpha, 1 / 2<\alpha<1$. Let $I_{h}: H^{s}(0,1) \cap H_{0}^{\alpha}(0,1) \rightarrow S_{h}$ be the interpolation operator. Then there exists a constant $C=C(\alpha)$ such that

$$
\left\|I_{h} v-v\right\|+h^{\alpha}\left\|I_{h} v-v\right\|_{H_{0}^{\alpha}(0,1)} \leq C h^{s}\|v\|_{H^{s}(0,1)} .
$$

Proof of Lemma 3.1: We first prove

$$
\left\|R_{h, \alpha} v-v\right\|_{H_{0}^{\alpha}(0,1)} \leq C h^{(r-\alpha)}\|v\|_{H^{r}(0,1)} .
$$

We have, noting that $B_{\alpha}\left(R_{h, \alpha} v-v, \chi\right)=0, \forall \chi \in S_{h}$,

$$
\begin{aligned}
& B_{\alpha}\left(R_{h, \alpha} v-v, R_{h, \alpha} v-v\right)=B_{\alpha}\left(R_{h, \alpha} v-v, R_{h, \alpha} v-\chi+\chi-v\right) \\
& =B_{\alpha}\left(R_{h, \alpha} v-v, \chi-v\right) \leq C\left\|R_{h, \alpha} v-v\right\|_{H_{0}^{\alpha}(0,1)}\|\chi-v\|_{H_{0}^{\alpha}(0,1)} .
\end{aligned}
$$

Note that, by Lemma 2.1,

$$
B_{\alpha}\left(R_{h, \alpha} v-v, R_{h, \alpha} v-v\right) \geq C\left\|R_{h, \alpha} v-v\right\|_{H_{0}^{\alpha}(0,1)}^{2} .
$$

We therefore get

$$
\left\|R_{h, \alpha} v-v\right\|_{H_{0}^{\alpha}(0,1)} \leq C\|\chi-v\|_{H_{0}^{\alpha}(0,1)}, \forall \chi \in H_{0}^{\alpha}(0,1),
$$

or

$$
\left\|R_{h, \alpha} v-v\right\|_{H_{0}^{\alpha}(0,1)} \leq C \inf _{\chi \in S_{h}}\|\chi-v\|_{H_{0}^{\alpha}(0,1)} \leq\left\|I_{h} v-v\right\|_{H_{0}^{\alpha}(0,1)} .
$$


Hence (24) follows by Lemma 3.2.

We next prove the following $L^{2}$-norm error estimates

$$
\left\|R_{h, \alpha} v-v\right\| \leq C h^{2(r-\alpha)}\|v\|_{H^{r}(0,1)} .
$$

Consider the following fractional elliptic problem, with $\varphi \in L^{2}(0,1)$,

$$
\begin{aligned}
& -{ }_{0}^{R} D_{x}^{2 \alpha} \psi(x)=\varphi, 0<x<1, \\
& \psi(0)=\psi(1)=0 .
\end{aligned}
$$

We have

$$
\begin{aligned}
\left(R_{h, \alpha} v-v, \varphi\right) & =\left(R_{h, \alpha} v-v,-{ }_{0}^{R} D_{x}^{2 \alpha} \psi\right)=-\left({ }_{x}^{R} D_{1}^{\alpha}\left(R_{h, \alpha} v-v\right),{ }_{0}^{R} D_{x}^{\alpha} \psi\right) \\
& \leq C\left\|R_{h, \alpha} v-v\right\|_{H_{0}^{\alpha}(0,1)}\|\psi-\chi\|_{H_{0}^{\alpha}(0,1)}, \forall \chi \in S_{h} .
\end{aligned}
$$

Therefore we have

$$
\left(R_{h, \alpha} v-v, \varphi\right) \leq C\left\|R_{h, \alpha} v-v\right\|_{H_{0}^{\alpha}(0,1)}\left\|\psi-I_{h} \psi\right\|_{H_{0}^{\alpha}(0,1)},
$$

where $I_{h}$ is the interpolation operator.

By (24), Lemma 3.2 and the regularity estimate (20), we obtain

$$
\left(R_{h, \alpha} v-v, \varphi\right) \leq\left(C h^{r-\alpha}\|v\|_{H^{r}(0,1)}\right)\left(C h^{r-\alpha}\|\psi\|_{H^{r}(0,1)}\right) \leq C h^{2(r-\alpha)}\|v\|_{H^{r}(0,1)}\|\varphi\| .
$$

Choose $\varphi=R_{h, \alpha} v-v$, we have $\left\|R_{h, \alpha} v-v\right\| \leq C h^{2(r-\alpha)}\|v\|_{H^{r}(0,1)}$.

Together these estimates complete the proof of Lemma 3.1.

We also need the following inverse estimates in $S_{h}$.

Lemma 3.3. Let $1 / 2<\alpha<1$. We have

$$
\|\chi\|_{H_{0}^{\alpha}(0,1)} \leq C h^{-\alpha}\|\chi\|, \quad \forall \chi \in S_{h} .
$$

Proof: Note that

$$
\|\chi\| \leq C h^{0}\|\chi\| \text { and }\|\chi\|_{H_{0}^{1}(0,1)} \leq C h^{-1}\|\chi\|, \quad \forall \chi \in S_{h} .
$$

By the moment inequality or interpolation inequality, with $\alpha_{1} \geq 0, \beta_{1} \geq 0, \gamma_{1} \geq 0$, with $A=-\frac{d^{2}}{d x^{2}}, \mathcal{D}(A)=$ $H^{2}(0,1) \cap H_{0}^{1}(0,1)$,

$$
\left\|A^{\alpha_{1}} u\right\| \leq C\left\|A^{\beta_{1}} u\right\|^{\theta}\left\|A^{\gamma_{1}} u\right\|^{1-\theta}, \quad \alpha_{1}=\theta \beta_{1}+(1-\theta) \gamma_{1}, 0 \leq \theta \leq 1,
$$

where $\theta=\frac{\alpha_{1}-\gamma_{1}}{\beta_{1}-\gamma_{1}}, 1-\theta=\frac{\beta_{1}-\alpha_{1}}{\beta_{1}-\gamma_{1}}$, Choosing $\alpha_{1}=\alpha / 2, \beta_{1}=0, \gamma_{1}=1 / 2$ and $\theta=1-\alpha, 1 / 2<\alpha<1$, we have

$$
\left\|A^{\alpha / 2} \chi\right\| \leq\left\|A^{0} \chi\right\|^{1-\alpha}\left\|A^{1 / 2} \chi\right\|^{\alpha},
$$

which implies that, with $1 / 2<\alpha \leq 1$, since $\left\|A^{\alpha / 2} \chi\right\|=\|\chi\|_{H_{0}^{\alpha}(0,1)},\left\|A^{1 / 2} \chi\right\|=|\chi|_{H_{0}^{1}(0,1)}$,

$$
\|\chi\|_{H_{0}^{\alpha}(0,1)} \leq C h^{-\alpha}\|\chi\|, \quad \forall \chi \in S_{h} .
$$

The proof of Lemma 3.3 is complete.

We next introduce the discrete fractional elliptic operator $A_{h, \alpha}: S_{h} \rightarrow S_{h}$ by, with $1 / 2<\alpha<1$,

$$
\left(A_{h, \alpha} \psi, \chi\right)=-\left({ }_{0}^{R} D_{x}^{\alpha} \psi,{ }_{x}^{R} D_{1}^{\alpha} \psi\right), \quad \forall \psi, \chi \in S_{h} .
$$


Lemma 3.4. Let $1 / 2<\alpha<1$. We have, with some constant $C=C(\alpha)$,

$$
\left\|A_{h, \alpha}\right\| \leq C h^{-2 \alpha},
$$

where $\|\cdot\|$ denotes the operator norm from $S_{h} \rightarrow S_{h}$.

Proof: For any $\psi \in S_{h}$, we have

$$
\left\|A_{h, \alpha} \psi\right\|=\sup _{\chi \in S_{h}} \frac{\left(A_{h, \alpha} \psi, \chi\right)}{\|\chi\|}=\sup _{\chi \in S_{h}} \frac{-\left({ }_{0}^{R} D_{x}^{\alpha} \psi,{ }_{x}^{R} D_{1}^{\alpha} \chi\right)}{\|\chi\|} \leq \frac{C\|\psi\|_{H_{0}^{\alpha}(0,1)}\|\chi\|_{H_{0}^{\alpha}(0,1)}}{\|\chi\|} .
$$

By Lemma 3.3, we have

$$
\left\|A_{h, \alpha} \psi\right\| \leq \frac{\left(C h^{-\alpha}\right)\|\psi\| \cdot\left(C h^{-\alpha}\right)\|\chi\|}{\|\chi\|} \leq C h^{-2 \alpha}\|\psi\|
$$

which implies (27). The proof of Lemma 3.4 is complete.

Let $E_{h, \alpha}(t)=e^{-A_{h, \alpha} t}$ be the semigroup generated by the operator $A_{h, \alpha}$. Then we have

Lemma 3.5. [21, Lemma 3.2] Let $0 \leq \gamma \leq 1$ and $1 / 2<\alpha<1$. There exists a constant $C=C(\alpha)$ such that, for $\chi \in S_{h}$,

$$
\left\|A_{h, \alpha}^{\gamma} E_{h, \alpha}(t) \chi\right\| \leq C t^{-\gamma}\|\chi\| .
$$

Theorem 3.6. Let $u_{h}$ and $u$ be the solutions of (16)-(17) and (12)-(13), respectively, where $B_{\alpha}(\cdot, \cdot)$ is defined by (14). Let $r=2 \alpha-1+\beta, 0 \leq \beta<1 / 2,1 / 2<\alpha<1$ and $u_{0} \in H^{r}(0,1)$. Assume that

$$
\left\|u_{0 h}-u_{0}\right\| \leq C h^{2(r-\alpha)}\left\|u_{0}\right\|_{H^{r}(0,1)} .
$$

Then, there exists a constant $C=C(\alpha, \beta)$ such that

$$
\max _{0 \leq t \leq T}\left\|u_{h}(t)-u(t)\right\| \leq C\left(1+\left|\log \left(T / h^{2 \alpha}\right)\right|\right) \max _{0 \leq t \leq T} h^{2(r-\alpha)}\|u(t)\|_{H^{r}(0,1)} .
$$

Proof: We write

$$
u_{h}(t)-u(t)=\theta(t)+\rho(t),
$$

where $\theta(t)=u_{h}(t)-R_{h, \alpha} u(t)$ and $\rho(t)=R_{h, \alpha} u(t)-u(t)$.

By Lemma 3.1, we have, with $r=2 \alpha-1+\beta, 0 \leq \beta<1 / 2,1 / 2<\alpha<1$,

$$
\|\rho(t)\| \leq C h^{2(r-\alpha)}\|u(t)\|_{H^{r}(0,1)} .
$$

We next consider the bound for $\theta(t)$. We will follow the ideas of the proof in Theorem 8.1 in [22] and apply a duality argument involving the following auxiliary problem: Given $t \in(0, T)$, let $\varphi_{h}:(0, t) \rightarrow S_{h}$ satisfy, with $\dot{\varphi}_{h}(s)=\frac{d \varphi_{h}(s)}{d s}$,

$$
\begin{aligned}
& -\left(\dot{\varphi}_{h}(s), \chi\right)+B_{\alpha}\left(\varphi_{h}(s), \chi\right)=0, \quad \forall \chi \in S_{h}, s \in(0, t), \\
& \varphi_{h}(t)=\theta(t) .
\end{aligned}
$$

Now taking $\chi=\theta(s)$ in (29), we have

$$
-\int_{0}^{t}\left(\dot{\varphi}_{h}(s), \theta(s)\right) d s+\int_{0}^{t} B_{\alpha}\left(\varphi_{h}(s), \theta(s)\right) d s=0 .
$$


Integrating by parts, we have

$$
\int_{0}^{t}\left(\varphi_{h}(s), \dot{\theta}(s)\right) d s-\left.\left(\varphi_{h}(s), \theta(s)\right)\right|_{s=0} ^{s=t}+\int_{0}^{t} B_{\alpha}\left(\varphi_{h}(s), \theta(s)\right) d s=0,
$$

or

$$
\|\theta(t)\|^{2}=\int_{0}^{t}\left[\left(\dot{\theta}(s), \varphi_{h}(s)\right)+B_{\alpha}\left(\theta(s), \varphi_{h}(s)\right)\right] d s+\left(\theta(0), \varphi_{h}(0)\right)
$$

Note that $\theta(s)=u_{h}(s)-u(s)-\rho(s)$ and

$$
\left(u_{h, s}(s)-u_{s}(s), \chi\right)+B_{\alpha}\left(u_{h}(s)-u(s), \chi\right)=0, \quad \forall \chi \in S_{h} .
$$

We have, noting that $B_{\alpha}(\rho(s), \chi)=0, \quad \forall \chi \in S_{h}, s \in(0, t)$,

$$
\begin{aligned}
\|\theta(t)\|^{2} & =\int_{0}^{t}\left[\left(-\dot{\rho}(s), \varphi_{h}(s)\right)+B_{\alpha}\left(-\rho(s), \varphi_{h}(s)\right)\right] d s \\
& +\left(u_{h}(0)-u(0), \varphi_{h}(0)\right)-\left(\rho(0), \varphi_{h}(0)\right) \\
& =\int_{0}^{t}\left(\rho(s), \dot{\varphi}_{h}(s)\right) d s-\left(\rho(t), \varphi_{h}(t)\right)+\left(u_{h}(0)-u(0), \varphi_{h}(0)\right)
\end{aligned}
$$

Thus, we have the following simple error representation formula

$$
\|\theta(t)\|^{2} \leq\left(\max _{0 \leq s \leq t}\|\rho(s)\|\right)\left(\int_{0}^{t}\left\|\dot{\varphi}_{h}(s)\right\| d s+\left\|\varphi_{h}(t)\right\|\right)+\left\|u_{h}(0)-u(0)\right\|\left\|\varphi_{h}(0)\right\| .
$$

We shall prove that

$$
\begin{aligned}
& \left\|\varphi_{h}(s)\right\| \leq C\|\theta(t)\|, \quad 0 \leq s \leq t \\
& \int_{0}^{t}\left\|\dot{\varphi}_{h}(s)\right\| d s \leq C\left(1+\left|\log \frac{t}{h^{2 \alpha}}\right|\right)\|\theta(t)\| .
\end{aligned}
$$

Assume that (32) and (33) hold at the moment, we then have, by (31),

$$
\|\theta(t)\| \leq C\left(1+\left|\log \frac{t}{h^{2 \alpha}}\right|\right) h^{2(r-\alpha)}\|u(t)\|_{H^{r}(0,1)} .
$$

It remains to show (32) and (33). Let $s=t-\bar{s}, \bar{s} \in(0, t)$. We have $\varphi_{h}(s)=\varphi_{h}(t-\bar{s})=\bar{\varphi}_{h}(\bar{s})$ and $\frac{d \bar{\varphi}_{h}(\bar{s})}{d \bar{s}}=\frac{d \varphi_{h}(t-\bar{s})}{d(t-\bar{s})}(-1)=-\frac{d \varphi_{h}(s)}{d s}$. Hence (29)-(30) are equivalent to the following initial value problem,

$$
\begin{aligned}
& \left(\dot{\bar{\varphi}}_{h}(\bar{s}), \chi\right)+B_{\alpha}\left(\bar{\varphi}_{h}(\bar{s}), \chi\right)=0, \quad \forall \chi \in S_{h}, s \in(0, t), \\
& \bar{\varphi}_{h}(0)=\theta(t) .
\end{aligned}
$$

Note that

$$
\left\|\varphi_{h}(s)\right\|=\left\|\bar{\varphi}_{h}(\bar{s})\right\| \text {, and } \int_{0}^{t}\left\|\dot{\varphi}_{h}(s)\right\| d s=\int_{0}^{t}\left\|\dot{\bar{\varphi}}_{h}(\bar{s})\right\| d \bar{s} .
$$

It is sufficient to show

$$
\begin{aligned}
& \left\|\bar{\varphi}_{h}(\bar{s})\right\| \leq C\|\theta(t)\|, \quad 0 \leq s \leq t, \\
& \int_{0}^{t}\left\|\dot{\bar{\varphi}}_{h}(s)\right\| d s \leq C\left(1+\left|\log \frac{t}{h^{2 \alpha}}\right|\right)\|\theta(t)\|,
\end{aligned}
$$


which we will prove now.

In fact, by using (26), we write (34) and (35) as the following abstract form

$$
\dot{\bar{\varphi}}_{h}(\bar{s})+A_{h, \alpha} \bar{\varphi}_{h}(\bar{s})=0, \quad \bar{\varphi}_{h}(0)=\theta(t) .
$$

By Lemmas 3.4 and 3.5, we have

$$
\left\|\bar{\varphi}_{h}(\bar{s})\right\|=\left\|E_{h, \alpha}(t) \bar{\varphi}_{h}(0)\right\| \leq C\left\|\bar{\varphi}_{h}(0)\right\| .
$$

Further we have

$$
\left\|\dot{\bar{\varphi}}_{h}(\bar{s})\right\|=\left\|-A_{h, \alpha} E_{h, \alpha}(t) \bar{\varphi}_{h}(0)\right\| \leq C \bar{s}^{-1}\left\|\bar{\varphi}_{h}(0)\right\| .
$$

Hence, by (39), (40) and Lemma 3.4

$$
\begin{aligned}
\int_{0}^{t}\left\|\dot{\varphi}_{h}(\bar{s})\right\| d \bar{s} & =\left[\int_{0}^{h^{2 \alpha}}+\int_{h^{2 \alpha}}^{t}\right]\left\|\dot{\bar{\varphi}}_{h}(\bar{s})\right\| d \bar{s}=\int_{0}^{h^{2 \alpha}}\left\|A_{h, \alpha} \bar{\varphi}_{h}(\bar{s})\right\| d \bar{s}+\int_{h^{2 \alpha}}^{t}\left\|\dot{\bar{\varphi}}_{h}(\bar{s})\right\| d \bar{s} \\
& \leq \int_{0}^{h^{2 \alpha}} h^{-2 \alpha}\left\|\bar{\varphi}_{h}(0)\right\| d \bar{s}+\int_{h^{2 \alpha}}^{t} \bar{s}^{-1}\left\|\bar{\varphi}_{h}(0)\right\| d \bar{s} \\
& \leq C\left(1+\log \left(\frac{t}{h^{2 \alpha}}\right)\right)\left\|\bar{\varphi}_{h}(0)\right\| .
\end{aligned}
$$

The proof of Theorem 3.6 is now complete.

\subsection{The two-sided space-fractional derivative case}

In this subsection, we will consider error estimates of the finite element methods for solving (12)-(13) in the two-sided space-fractional derivative case. To do this, we need to consider the two-sided fractional elliptic problem (10)-(11).

Lemma 3.7. Let $1 / 2<\alpha<1$. The solution of (10)-(11) takes the form of

$$
w(x)=c_{1}+c_{2} K_{1 / 2}(x)+F(x),
$$

where $c_{1}=-F(0)$ and $c_{2}=-\frac{F(1)+c_{1}}{K_{1 / 2}(1)}$ and $F(x)$ is some smooth function such that $\mathcal{L}^{2 \alpha} F(x)=g(x)$.

Proof: Since $w(0)=0$, we have

$$
\begin{aligned}
{ }_{0}^{R} D_{x}^{2 \alpha} w(x) & =D_{0}^{2}{ }_{0}^{R} D_{x}^{2 \alpha-2} w(x)=D\left(D_{0}^{R} D_{x}^{2 \alpha-2} w(x)\right) \\
& =D\left({ }_{0}^{R} D_{x}^{2 \alpha-2} D w(x)\right)=\left(D_{0}^{R} D_{x}^{2 \alpha-2} D\right) w(x) .
\end{aligned}
$$

Similarly we have, since $w(1)=0$,

$$
{ }_{x}^{R} D_{1}^{2 \alpha} w(x)=\left(D_{x}^{R} D_{1}^{2 \alpha-2} D\right) w(x) .
$$

Thus the operator $\mathcal{L}^{2 \alpha}$ can be written as, noting that $w(0)=w(1)=0$,

$$
\mathcal{L}^{2 \alpha} w(x)=-\frac{1}{2}\left(D_{0}^{R} D_{x}^{2 \alpha-2} D+D_{0}^{R} D_{x}^{2 \alpha-2} D\right) w(x), 0<x<1 .
$$

By Ervin et al. $[9,(4.8)]$, we know that the kernel of $\mathcal{L}^{2 \alpha}$ is

$$
K_{1 / 2}(x)=\frac{1}{\alpha} x_{2}^{\alpha} F_{1}(\alpha, 1-\alpha ; 1+\alpha, x),
$$

that is, $\mathcal{L}^{2 \alpha} K_{1 / 2}(x)=0$. Here ${ }_{2} F_{1}(a, b ; c, x)$ is so called Gaussian three-parameter hyper-geometric function defined as in Ervin et al. [9, Definition 1]. Assume that $F(x)$ is some smooth function such that $\mathcal{L}^{2 \alpha} F(x)=$ $g(x)$. Then it is easy to check that the solution of (10)-(11) has the form (41) as required.

The proof of Lemma 3.7 is now complete. 
Remark 3.2. In general it may be difficult to find the smooth solution $F(x)$ such that $\mathcal{L}^{2 \alpha} F(x)=g(x), 0<$ $x<1$. Below is an example for the special $g(x)=-\frac{1}{2} \frac{1}{\Gamma(2-2 \alpha)} x^{1-2 \alpha}+\frac{1}{2} \frac{1}{\Gamma(2-2 \alpha)}(1-x)^{1-2 \alpha}$. In this case, it is easy to see that, with $F(x)=1+x$,

$$
\mathcal{L}^{2 \alpha} F(x)=-\frac{1}{2} \frac{1}{\Gamma(2-2 \alpha)} x^{1-2 \alpha}+\frac{1}{2} \frac{1}{\Gamma(2-2 \alpha)}(1-x)^{1-2 \alpha} .
$$

Hence the solution of (10)-(11) is

$$
w(x)=-1-\left(K_{1 / 2}(1)\right)^{-1} K_{1 / 2}(x)+(1+x)=x-\left(K_{1 / 2}(1)\right)^{-1} K_{1 / 2}(x) .
$$

We will come back to this example in Example 5.5 in Section 5.

Note that $x^{\alpha} \in \tilde{H}_{L}^{\alpha+\beta}(0,1), 0<\beta<1 / 2,1 / 2<\alpha<1$, see Jin et al. [20]. Thus, by $(42), K_{1 / 2} \in$ $\tilde{H}_{L}^{\alpha+\beta}(0,1)$ which implies that the solution $w$ of $(10)-(11)$ satisfies $w \in \tilde{H}_{L}^{\alpha+\beta}(0,1)$. Hence for the general $g \in L^{2}(0,1)$, we can not guarantee the solution of (10)-(11) satisfies $w \in H^{2 \alpha}(0,1)$. Recall that when $\alpha=1$, the equation (10)-(11) reduces to the standard elliptic equation and it is well-known that $w \in H^{2 \alpha}(0,1)=$ $H^{2}(0,1)$ in this case.

Following the argument for the formulation of (19), we may write (10)-(11) as the following abstract form, with $g \in L^{2}(0,1)$,

$$
A_{\alpha} w=g
$$

Assume that $g$ is sufficiently regular such that the regularity of $w$ is determined by the kernel of $\mathcal{L}^{2 \alpha}, 1 / 2<$ $\alpha<1$, we may have the following regularity property, see [9, Section 5],

$$
\|w\|_{\tilde{H}_{L}^{\alpha+\beta}(0,1)} \leq\left\|A_{\alpha} w\right\|_{L^{2}(0,1)}=\|g\|_{L^{2}(0,1)} .
$$

Theorem 3.8. Let $u_{h}$ and $u$ be the solutions of (16)-(17) and (12)-(13), respectively, where $B_{\alpha}(\cdot, \cdot)$ is defined by (15). Let $1 / 2<\alpha<1$ and $u_{0} \in H^{\alpha+\beta}(0,1), 0<\beta<1 / 2$. Assume that

$$
\left\|u_{0 h}-u(0)\right\| \leq C h^{2 \beta}\left\|u_{0}\right\|_{H^{\alpha+\beta}(0,1)} .
$$

Then, there exists a constant $C=C(\alpha)$ such that

$$
\max _{0 \leq t \leq T}\left\|u_{h}(t)-u(t)\right\| \leq C\left(1+\left|\log \left(T / h^{2 \alpha}\right)\right|\right) \max _{0 \leq t \leq T} h^{2 \beta}\|u(t)\|_{H^{\alpha+\beta}(0,1)}
$$

The proof of Theorem 3.8 is similar to the proof of Theorem 3.6. We also need to introduce the corresponding fractional Ritz projection operator $R_{h, \alpha}: H_{0}^{\alpha}(0,1) \rightarrow S_{h}, 1 / 2<\alpha<1$ and the discrete fractional elliptic operator $A_{h, \alpha}: S_{h} \rightarrow S_{h}$. In this case the discrete fractional elliptic operator is symmetric, positive definite. Therefore we may use the spectral expansion of the operator to prove the following smoothing property of the semigroup $E_{h, \alpha}(t)$ : with $0 \leq \gamma \leq 1,1 / 2<\alpha<1$,

$$
\left\|A_{h, \alpha}^{\gamma} E_{h, \alpha}(t) \chi\right\| \leq C t^{-\gamma}\|\chi\|
$$

which we need in the proof of Theorem 3.8 .

\section{Error estimates proved by using the approximate solution operators}

\subsection{The left-sided Riemann-Liouville fractional derivative case}

In this subsection, we will prove the error estimates of the finite element methods for solving (12)-(13) by using the approximate solution operators in the left-sided Riemann-Liouville fractional derivative case, see [38, Chapter 2] 
Let us consider, with $1 / 2<\alpha<1, f \in L^{2}(0,1)$,

$$
-{ }_{0}^{R} D_{x}^{2 \alpha} u(x)=f(x), \quad 0<x<1, \quad \text { with } u(0)=u(1)=0 .
$$

We recall that the equation (46) can be written as the following operator form

$$
A_{\alpha} u=f,
$$

where $A_{\alpha}: \mathcal{D}\left(A_{\alpha}\right) \rightarrow L^{2}(0,1)$ is defined in (19).

The variational form of $(46)$ is to find $u \in H_{0}^{\alpha}(0,1)$ such that

$$
B_{\alpha}(u, v)=(f, v), \quad \forall v \in H_{0}^{\alpha}(0,1) .
$$

By regularity estimate (20) for the fractional elliptic problem, the equation (46) or (47) has a unique solution $u \in H^{r}(0,1) \cap H_{0}^{\alpha}(0,1)$, where $r=2 \alpha-1+\beta, 0 \leq \beta<1 / 2,1 / 2<\alpha<1$. Denote $T_{\alpha}: L^{2}(0,1) \rightarrow$ $H^{r}(0,1) \cap H_{0}^{\alpha}(0,1)$ the solution operator of $(47)$, i.e.,

$$
u=T_{\alpha} f, \quad f \in L^{2}(0,1) .
$$

We then have

Lemma 4.1. Let $T_{\alpha}: L^{2}(0,1) \rightarrow H^{r}(0,1) \cap H_{0}^{\alpha}(0,1), r=2 \alpha-1+\beta, 0 \leq \beta<1 / 2,1 / 2<\alpha<1$ be the solution operator of (47). We have

$$
\begin{aligned}
& T_{\alpha}\left(A_{\alpha} u\right)=u, \quad \forall u \in H^{r}(0,1) \cap H_{0}^{\alpha}(0,1), \\
& A_{\alpha} T_{\alpha} f=f, \quad \forall f \in L^{2}(0,1) .
\end{aligned}
$$

In other words, we have $T_{\alpha}=A_{\alpha}^{-1}$.

Proof: Assume that $u$ is the solution of (47), i.e., $u=T_{\alpha} f$ and

$$
B_{\alpha}(u, v)=(f, v), \quad \forall v \in H_{0}^{\alpha}(0,1) .
$$

Further assume that $A_{\alpha} u=f$. Then we have

$$
T_{\alpha}\left(A_{\alpha} u\right)=u, \quad \forall u \in H^{r}(0,1) \cap H_{0}^{\alpha}(0,1) .
$$

On the other hand, noting that $u=T_{\alpha} f$ satisfies

$$
B_{\alpha}\left(T_{\alpha} f, v\right)=(f, v), \quad \forall v \in H_{0}^{\alpha}(0,1),
$$

we have, by the regularity estimate (20),

$$
\left(A_{\alpha}\left(T_{\alpha} f\right), v\right)=(f, v), \quad \forall v \in H_{0}^{\alpha}(0,1),
$$

which implies that

$$
A_{\alpha} T_{\alpha} f=f, \quad \forall f \in L^{2}(0,1) .
$$

Next we introduce the approximate solution operator $T_{h, \alpha}: L^{2}(0,1) \rightarrow S_{h}$ for the finite element approximation problem of (47). Recall that the finite element problem of (47) is to find $u_{h} \in S_{h}$ such that

$$
B_{\alpha}\left(u_{h}, \chi\right)=(f, \chi), \quad \forall \chi \in S_{h} .
$$

Denote $T_{h, \alpha}: L^{2}(0,1) \rightarrow S_{h}$ the solution operator of (49), i.e.,

$$
u_{h}=T_{h, \alpha} f .
$$


Lemma 4.2. Let $T_{h, \alpha}: L^{2}(0,1) \rightarrow S_{h}$ be the solution operator of (49). We have

$$
T_{h, \alpha} A_{h, \alpha} u_{h}=u_{h} .
$$

Further if $f_{h} \in S_{h}$, we have

$$
A_{h, \alpha} T_{h, \alpha} f_{h}=f_{h}, \quad \forall f_{h} \in S_{h}
$$

Proof: By (49), we have

$$
B_{\alpha}\left(u_{h}, \chi\right)=(f, \chi), \quad \forall \chi \in S_{h},
$$

or

$$
\left(A_{h, \alpha} u_{h}, \chi\right)=(f, \chi)=\left(P_{h} f, \chi\right), \quad \forall \chi \in S_{h} .
$$

Here $P_{h}: L^{2}(0,1) \rightarrow S_{h}$ is the $L^{2}$ projection operator on $S_{h}$. Hence we have

$$
A_{h, \alpha} u_{h}=P_{h} f,
$$

which implies that

$$
T_{h, \alpha} A_{h, \alpha} u_{h}=T_{h, \alpha} P_{h} f .
$$

Note that

$$
T_{h, \alpha} P_{h} f=T_{h, \alpha} f,
$$

which we will prove later. Assume this is true at the moment, we have

$$
T_{h, \alpha} A_{h, \alpha} u_{h}=T_{h, \alpha} P_{h} f=T_{h, \alpha} f=u_{h},
$$

which is (50).

We now show (52). Note that $B_{\alpha}\left(T_{h, \alpha} P_{h} f, \chi\right)=\left(P_{h} f, \chi\right)$, and $B_{\alpha}\left(T_{h, \alpha} f, \chi\right)=(f, \chi)$, we have

$$
B_{\alpha}\left(T_{h, \alpha} P_{h} f-T_{h, \alpha} f, \chi\right)=0,
$$

which implies that

$$
\left\|T_{h, \alpha} P_{h} f-T_{h, \alpha} f\right\|_{H_{0}^{\alpha}(0,1)}=0 .
$$

By Lemma 2.4, we have

$$
\left\|T_{h, \alpha} P_{h} f-T_{h, \alpha} f\right\|_{L^{2}(0,1)} \leq\left\|T_{h, \alpha} P_{h} f-T_{h, \alpha} f\right\|_{H_{0}^{\alpha}(0,1)}=0,
$$

which implies that

$$
T_{h, \alpha} P_{h} f=T_{h, \alpha} f, \quad \forall f \in L^{2}(0,1) .
$$

To show (51), we note that $u_{h}=T_{h, \alpha} f_{h}$ satisfies

$$
B_{\alpha}\left(T_{h, \alpha} f_{h}, \chi\right)=\left(f_{h}, \chi\right), \quad \forall \chi \in S_{h},
$$

that is

$$
\left(A_{h, \alpha} T_{h} f_{h}, \chi\right)=\left(f_{h}, \chi\right), \quad \forall \chi \in S_{h},
$$

which implies that

$$
A_{h, \alpha} T_{h, \alpha} f_{h}=f_{h} .
$$

Hence we show $T_{h, \alpha}=A_{h, \alpha}^{-1}$ on $S_{h}$.

Together these estimates complete the proof of this lemma.

Lemma 4.3. Let $T_{h, \alpha}$ and $T_{\alpha}$ be the solution operators of (49) and (47), respectively. Let $r=2 \alpha-1+\beta, 0 \leq$ $\beta<1 / 2,1 / 2<\alpha<1$. Then, there exists a constant $C=C(\alpha, \beta)$ such that

$$
\left\|u_{h}-u\right\|=\left\|\left(T_{h, \alpha}-T\right) f\right\| \leq C h^{2(r-\alpha)}\|f\| .
$$


Proof: We first show that $T_{h, \alpha}=R_{h, \alpha} T_{\alpha}$, where $R_{h, \alpha}: H_{0}^{\alpha}(0,1) \rightarrow S_{h}$ is the Ritz projection operator defined by (21)

In fact, we have

$$
B_{\alpha}\left(T_{h, \alpha} f, \chi\right)=(f, \chi)=B_{\alpha}\left(T_{\alpha} f, \chi\right)=B_{\alpha}\left(R_{h, \alpha} T_{\alpha} f, \chi\right), \quad \forall \chi \in S_{h} .
$$

Thus

$$
B_{\alpha}\left(T_{h, \alpha} f-R_{h, \alpha} T_{\alpha} f, \chi\right)=0, \quad \forall \chi \in S_{h},
$$

which implies that $T_{h, \alpha}=R_{h, \alpha} T_{\alpha}$.

Thus we have, by Lemma 3.1,

$$
\left\|\left(T_{h, \alpha}-T_{\alpha}\right) f\right\|=\left\|\left(R_{h, \alpha} T_{\alpha}-T_{\alpha}\right) f\right\| \leq C h^{2(r-\alpha)}\left\|T_{\alpha} f\right\|_{H^{r}(0,1)} \leq C h^{2(r-\alpha)}\|f\| .
$$

We now use the solution operators $T_{\alpha}$ and $T_{h, \alpha}$ to consider the error estimates of the space-fractional partial differential equations (12)-(13). The equations (12)-(13) can be written into the following abstract form

$$
u_{t}+A_{\alpha} u=f, t>0 \quad \text { with } u(0)=u_{0} .
$$

Note that $T_{\alpha}=A_{\alpha}^{-1},(53)$ is equivalent to

$$
T_{\alpha} u_{t}+u=T_{\alpha} f, t>0 \quad \text { with } u(0)=u_{0} .
$$

The finite element method of (53) is to find $u_{h}(t) \in S_{h}$ such that

$$
\begin{aligned}
& \left(u_{h, t}, \chi\right)+B_{\alpha}\left(u_{h}, \chi\right)=(f, \chi), t>0 \quad \forall \chi \in S_{h}, \\
& u_{h}(0)=u_{0 h},
\end{aligned}
$$

which can be written into the abstract form

$$
u_{h, t}+A_{h, \alpha} u_{h}=P_{h} f, t>0 \quad \text { with } u_{h}(0)=u_{0 h} .
$$

Note that $T_{h, \alpha}=A_{h, \alpha}^{-1},(57)$ is equivalent to

$$
T_{h, \alpha} u_{h, t}+u_{h}=T_{h, \alpha} P_{h} f, t>0 \quad \text { with } u_{h}(0)=u_{0 h} .
$$

We then have the following error estimates

Theorem 4.4. Let $u_{h}$ and $u$ be the solutions of (58) and (54), respectively. Let $r=2 \alpha-1+\beta, 0 \leq \beta<$ $1 / 2,1 / 2<\alpha<1$. Assume that $u_{0} \in H^{r}(0,1)$. Then, there exists a constant $C=C(\alpha, \beta)$ such that

$$
\left\|u_{h}(t)-u(t)\right\| \leq\left\|u_{0 h}-u(0)\right\|+C h^{2(r-\alpha)}\left(\left\|u_{0}\right\|_{H^{r}(0,1)}+\int_{0}^{t}\left\|u_{t}(s)\right\|_{H^{r}(0,1)} d s\right) .
$$

Proof: Let $e=u_{h}-u$. Then $e$ satisfies the error equation

$$
\begin{aligned}
T_{h, \alpha} e_{t}+e & =T_{h, \alpha}\left(u_{h, t}-u_{t}\right)+\left(u_{h}-u\right)=\left(T_{h, \alpha} u_{h, t}+u_{h}\right)-\left(T_{h, \alpha} u_{t}+u\right) \\
& =T_{h, \alpha} f-\left(T_{h, \alpha} u_{t}-T_{\alpha} u_{t}+T_{\alpha} u_{t}+u\right) \\
& =\left(T_{h, \alpha}-T_{\alpha}\right) f-\left(T_{h, \alpha}-T_{\alpha}\right) u_{t}=\left(T_{h, \alpha}-T_{\alpha}\right)\left(f-u_{t}\right) \\
& =\left(T_{\alpha}-T_{h, \alpha}\right)\left(-A_{\alpha} u\right) .
\end{aligned}
$$

Note that $T_{h, \alpha}=R_{h, \alpha} T_{\alpha}$, we have, with $T_{\alpha} A_{\alpha}=I$,

$$
T_{h, \alpha} e_{t}+e=\underset{14}{\left(R_{h, \alpha}-I\right) u=\rho .}
$$


Following the same argument as in Thomée [38, p. 33, Theorem 2.3], we have

$$
\begin{aligned}
\|e(t)\| & \leq\|e(0)\|+C\left(\|\rho(0)\|+\int_{0}^{t}\left\|\rho_{t}(s)\right\| d s\right) \\
& =\left\|u_{0 h}-u_{0}\right\|+C h^{2(r-\alpha)}\left(\|u(0)\|_{H^{r}(0,1)}+\int_{0}^{t}\left\|u_{t}(s)\right\|_{H^{r}(0,1)} d s\right) .
\end{aligned}
$$

The proof of Theorem 4.4 is now complete.

\subsection{The two-sided space-fractional derivative case}

In this subsection, we will consider the error estimates of the finite element methods for solving (12)(13) by using the approximate solution operators in the two-sided space-fractional derivative case, see [38, Chapter 2].

We may introduce the corresponding solution operator $T_{\alpha}$ and $T_{h, \alpha}$ as in Section 4.1. In this case, $T_{\alpha}: L^{2}(0,1) \rightarrow H^{r}(0,1) \cap H_{0}^{\alpha}, \alpha \leq r \leq 2 \alpha, 1 / 2<\alpha<1$ is a selfadjoint and positive definite operator on $L^{2}(0,1)$ and $T_{\alpha, h}: L^{2}(0,1) \rightarrow S_{h}$ is a selfadjoint and semi-positive definite operator on $L^{2}(0,1)$ and positive definite on $S_{h}$. Then, the equations (12)-(13) can be written as the following abstract form

$$
T_{\alpha} u_{t}+u=T_{\alpha} f, t>0 \quad \text { with } u(0)=u_{0},
$$

and the finite element method of (59) can be written as

$$
T_{h, \alpha} u_{h, t}+u_{h}=T_{h, \alpha} P_{h} f, t>0 \quad \text { with } u_{h}(0)=u_{0 h} .
$$

We have

Theorem 4.5. Let $u_{h}$ and $u$ be the solutions of (60) and (59), respectively. Let $\alpha \leq r \leq 2 \alpha, 1 / 2<\alpha<1$. Assume that $u_{0} \in H^{r}(0,1)$. Then there exists a constant $C=C(\alpha)$ such that

$$
\left\|u_{h}(t)-u(t)\right\| \leq\left\|u_{0 h}-u(0)\right\|+C h^{2(r-\alpha)}\left(\left\|u_{0}\right\|_{H^{r}(0,1)}+\int_{0}^{t}\left\|u_{t}(s)\right\|_{H^{r}(0,1)} d s\right) .
$$

Proof: The Proof of Theorem 4.5 is similar to the proof of Theorem 4.4. We omit the proof here.

\section{Numerical simulations}

In this section, we will consider some numerical examples for solving space-fractional partial differential equation (1)-(3) or (4)-(6).

Let $0=x_{0}<x_{1}<\cdots<x_{N_{h}+1}=1$ be a partition of the space interval $[0,1]$ and $h$ the space step size. Let $S_{h} \subset H_{0}^{\alpha}(0,1)$ be the continuous piecewise linear finite element space. Let $0=t_{0}<t_{1}<\cdots<t_{n}<$ $\cdots<t_{N}=T$ be a partition of time interval $[0, T]$ and $\Delta t$ be the time step size. The implicit Euler method of (16)-(17) is to find $U^{n} \approx u_{h}\left(t_{n}\right)$, such that

$$
\begin{aligned}
& \left(U^{n}, \chi\right)+\Delta t B_{\alpha}\left(U^{n}, \chi\right)=\Delta t(f, \chi)+\left(U^{n-1}, \chi\right), \quad \forall \chi \in S_{h}, \\
& U^{0}=u_{0 h} .
\end{aligned}
$$

Let

$$
U^{n}=\sum_{\substack{j=1 \\ 15}}^{N_{h}} \alpha_{j}^{n} \varphi_{j}
$$


where $\left\{\varphi_{1}, \varphi_{2}, \ldots \varphi_{N_{h}}\right\}$ are the finite element basis functions in $S_{h}$. We then have, with $\chi=\varphi_{l}, l=$ $1,2, \ldots, N_{h}$,

$$
\sum_{j=1}^{N_{h}}\left(\varphi_{j}, \varphi_{l}\right) \alpha_{j}^{n}+\Delta t \sum_{j=1}^{N_{h}} B_{\alpha}\left(\varphi_{j}, \varphi_{l}\right)=\Delta t\left(f, \varphi_{l}\right)+\sum_{j=1}^{N_{h}} \alpha_{j}^{n-1}\left(\varphi_{j}, \varphi_{l}\right) .
$$

Denote $\mathbf{M}=\left(\varphi_{j}, \varphi_{l}\right)_{j, l=1}^{N_{h}}, \quad \mathbf{S}_{\alpha}=\left(B_{\alpha}\left(\varphi_{j}, \varphi_{l}\right)\right)_{j, l=1}^{N_{h}}, \quad \mathbf{F}=\left(f, \varphi_{l}\right)_{l=1}^{N_{h}}$ and $\alpha^{n}=\left(\alpha_{j}^{n}\right)_{j=1}^{N_{h}}$. We get the following matrix form

$$
\left(\mathbf{M}+\Delta t \cdot \mathbf{S}_{\alpha}\right) \alpha^{n}=\Delta t \mathbf{F}+\mathbf{M} \alpha^{n-1}, n=1,2,3, \ldots
$$

where $\alpha^{0}$ is obtained by $U^{0}=\sum_{j=1}^{N_{h}} \alpha_{j}^{0} \varphi_{j}$.

To obtain the matrix $\mathbf{S}_{\alpha}$, we shall use the following relations, with $v^{\prime}=\frac{d v}{d x}$,

$$
\left({ }_{0}^{R} D_{x}^{\alpha} \varphi_{j},{ }_{x}^{R} D_{1}^{\alpha} \varphi_{l}\right)=\left({ }_{0}^{R} D_{x}^{2 \alpha-1} \varphi_{j}, \varphi_{l}^{\prime}\right), j, l=1,2, \ldots, N_{h},
$$

and

$$
\left({ }_{x}^{R} D_{1}^{\alpha} \varphi_{j},{ }_{0}^{R} D_{x}^{\alpha} \varphi_{l}\right)=\left({ }_{x}^{R} D_{1}^{2 \alpha-1} \varphi_{j}, \varphi_{l}^{\prime}\right), j, l=1,2, \ldots, N_{h},
$$

which can be calculated exactly by using the following formulas, with $j=1,2,3, \ldots, N_{h}$,

$$
{ }_{0}^{R} D_{x}^{\alpha} \varphi_{j}= \begin{cases}0, \quad 1 & 0<x<x_{j-1}, \\ \frac{1}{h \Gamma(2-\alpha)}\left(x-x_{j-1}\right)^{1-\alpha}, & x_{j-1}<x<x_{j}, \\ \frac{1}{h \Gamma(2-\alpha)}\left[\left(x-x_{j-1}\right)^{1-\alpha}-2\left(x-x_{j}\right)^{1-\alpha}\right], & x_{j}<x<x_{j+1}, \\ \frac{1}{h \Gamma(2-\alpha)}\left[\left(x-x_{j-1}\right)^{1-\alpha}-2\left(x-x_{j}\right)^{1-\alpha}+\left(x-x_{j+1}\right)^{1-\alpha}\right], & x_{j+1}<x<1,\end{cases}
$$

and

$$
{ }_{x}^{R} D_{1}^{\alpha} \varphi_{l}= \begin{cases}\frac{1}{h \Gamma(2-\alpha)}\left[-\left(x_{l+1}-x\right)^{1-\alpha}+2\left(x_{l}-x\right)^{1-\alpha}-\left(x_{l-1}-x\right)^{1-\alpha}\right], & 0<x<x_{l-1}, \\ \frac{1}{h \Gamma(2-\alpha)}\left[-\left(x_{l+1}-x\right)^{1-\alpha}+2\left(x_{l}-x\right)^{1-\alpha}\right], & x_{l-1}<x<x_{l}, \\ \frac{1}{h \Gamma(2-\alpha)}\left[-\left(x_{l+1}-x\right)^{1-\alpha}\right], & x_{l}<x<x_{l+1}, \\ 0, & x_{l+1}<x<1 .\end{cases}
$$

Example 5.1. [21] Consider the following linear space-fractional partial differential equation, with $1 / 2<$ $\alpha<1$,

$$
\begin{aligned}
& \frac{\partial u(x, t)}{\partial t}-{ }_{0}^{R} D_{x}^{2 \alpha} u(x, t)=f(x, t), \quad 0<t<T, 0<x<1, \\
& u(0, t)=u(1, t)=0, \quad 0<t<T, \\
& u(x, 0)=u_{0}(x), \quad 0<x<1,
\end{aligned}
$$

where $u_{0}(x)=x(1-x)$ and $f(x, t)=0$.

We will consider the experimentally determined orders of convergence ("EOC") of $\left\|u\left(t_{N}\right)-U^{N}\right\|$ for the different $\alpha$ at $t_{N}=1$. We choose $\Delta t=0.001$ and let $e_{N}^{(i)}=\left\|u\left(t_{N}\right)-U^{N}\right\|$ denote the $L^{2}$ norm at $t_{N}=1$ obtained by using the different space step sizes $h_{i}$. Since the exact solution is not available in this example, we calculate the reference solution ( or 'true' solution) $u\left(t_{N}\right)$ by using the very small time step size $\Delta t=0.00001$ and space step size $h=2^{-10}$. By Theorem 3.6, we have, with some $r=2 \alpha-1+\beta, 0 \leq \beta<1 / 2,1 / 2<\alpha<1$,

$$
e_{N}^{(i)} \leq C h_{i}^{2(r-\alpha)}
$$


which implies that the theoretical convergence order $2(r-\alpha)$ satisfies

$$
\log _{2}\left(e_{N}^{(i)} / e_{N}^{(i+1)}\right) \approx \log _{2}\left(h_{i} / h_{i+1}\right)^{2(r-\alpha)}=2(r-\alpha)
$$

The best theoretical convergence order is approximately $2 \alpha-1$ when $\beta \approx 1 / 2$. For the fixed $\alpha$, in Table 1 , we calculate the experimentally determined orders of convergence with respect to the different space step sizes $h_{i}=1 / 2^{i}, i=4,5,6,7$, where the numbers in the brackets are the theoretical convergence orders. The numerical results in Table 1 are consistent with the numerical results in Jin et al. [21, Table 1].

\begin{tabular}{cccccc}
\hline$\alpha$ & $h=1 / 16$ & $h=1 / 32$ & $h=1 / 64$ & $\mathrm{~h}=1 / 128$ & (theoretical orders) \\
\hline $5 / 8$ & $1.38 \mathrm{e}-3$ & $7.76 \mathrm{e}-4$ & $4.87 \mathrm{e}-4$ & $3.39 \mathrm{e}-4$ & \\
& & 0.83 & 0.67 & 0.52 & $(0.25)$ \\
$3 / 4$ & $7.52 \mathrm{e}-5$ & $3.47 \mathrm{e}-5$ & $1.70 \mathrm{e}-5$ & $9.46 \mathrm{e}-5$ & \\
& & 1.11 & 1.03 & 0.84 & $(0.50)$ \\
$7 / 8$ & $1.22 \mathrm{e}-6$ & $5.45 \mathrm{e}-7$ & $2.24 \mathrm{e}-7$ & $8.59 \mathrm{e}-8$ & \\
& & 1.16 & 1.28 & 1.38 & $(0.75)$ \\
\hline
\end{tabular}

Table 1: The experimentally determined orders of convergence ("EOC") for the different $\alpha$ at $t_{N}=1$ in Example 5.1

Example 5.2. Consider the following linear space-fractional partial differential equation, with $1 / 2<\alpha<1$,

$$
\begin{aligned}
& \frac{\partial u(x, t)}{\partial t}-{ }_{0}^{R} D_{x}^{2 \alpha} u(x, t)=f(x, t), \quad 0<t<T, 0<x<1, \\
& u(0, t)=u(1, t)=0, \quad 0<t<T, \\
& u(x, 0)=u_{0}(x), \quad 0<x<1,
\end{aligned}
$$

where $u_{0}(x)=0$ and $f(x, t)=t x^{1 / 4}$.

In this example, we choose $f(x, t)=t x^{1 / 4}$, where $x^{1 / 4} \in H^{1 / 4-\epsilon}$ for any $\epsilon>0$ and $f \in L^{2}(0, T ; H)$. By Theorems 3.6, we have, with some $r=2 \alpha-1+\beta, 0 \leq \beta<1 / 2,1 / 2<\alpha<1$,

$$
e_{N}^{(i)} \leq C h_{i}^{2(r-\alpha)}
$$

We use the same notations as in Example 5.1. In Table 2, we observe that the experimentally determined orders of convergence ("EOC") are also higher than the theoretical one, where the numbers in the brackets are the theoretical convergence orders.

\begin{tabular}{ccccccc}
\hline$\alpha$ & $h=1 / 4$ & $h=1 / 8$ & $h=1 / 16$ & $\mathrm{~h}=1 / 32$ & $\mathrm{~h}=1 / 64$ & (theoretical orders) \\
\hline $5 / 8$ & $7.70 \mathrm{e}-2$ & $4.86 \mathrm{e}-2$ & $2.97 \mathrm{e}-2$ & $1.81 \mathrm{e}-2$ & $1.17 \mathrm{e}-2$ & \\
& & 0.66 & 0.71 & 0.71 & 0.63 & $(0.25)$ \\
$3 / 4$ & $1.99 \mathrm{e}-2$ & $9.85 \mathrm{e}-3$ & $4.89 \mathrm{e}-3$ & $2.43 \mathrm{e}-3$ & $1.22 \mathrm{e}-3$ & \\
& & 1.01 & 1.01 & 1.01 & 1.00 & $(0.50)$ \\
$7 / 8$ & $3.63 \mathrm{e}-3$ & $1.38 \mathrm{e}-3$ & $5.47 \mathrm{e}-4$ & $2.21 \mathrm{e}-4$ & $8.88 \mathrm{e}-5$ & \\
& & 1.39 & 1.34 & 1.31 & 1.32 & $(0.75)$ \\
\hline
\end{tabular}

Table 2: The experimentally determined orders of convergence ("EOC") for the different $\alpha$ at $t_{N}=1$ in Example 5.2 
Example 5.3. [48] Consider the following linear space-fractional partial differential equation, with $1 / 2<$ $\alpha<1$,

$$
\begin{aligned}
& \frac{\partial u(x, t)}{\partial t}-{ }_{0}^{R} D_{x}^{2 \alpha} u(x, t)=f(x, t), \quad 0<t<T, 0<x<1, \\
& u(0, t)=u(1, t)=0, \quad 0<t<T, \\
& u(x, 0)=u_{0}(x), \quad 0<x<1,
\end{aligned}
$$

where $u_{0}(x)=10 x^{2}(1-x)^{2}$ and

$$
f(x, t)=-10 e^{-t} x^{2}(1-x)^{2}-\frac{10 e^{-t} x^{2-2 \alpha}}{\Gamma(3-2 \alpha)}\left(2-\frac{12 x}{3-2 \alpha}+\frac{24 x^{2}}{(3-2 \alpha)(4-2 \alpha)}\right) .
$$

In this example, we have a very smooth exact solution $u(x, t)=10 e^{-t} x^{2}(1-x)^{2}$ and $u \in L^{2}\left(0, T ; H^{2}(0,1)\right)$. In Table 3, we observe that the experimentally determined order of convergence ("EOC") is much higher than the order in Example 5.2 for the different $\alpha \in(1 / 2,1)$.

To see the efficiency of the finite element method for solving space-fractional partial differential equations, we shall compare the finite element method with the popular Grünwald-Letnikov (G-L) finite difference method introduced in [28] and [29] which has the convergence order $O(h)$. We see that, in Table 3 the convergence order of the finite element method (FEM) is much higher than the convergence order of the Grünwald-Letnikov (G-L) finite difference method in [28] and [29]. Another advantage of using finite element method for solving space-fractional partial differential equation is that there is no restriction for the boundary conditions of the space-fractional partial differential equation. However the Grünwald-Letnikov finite difference methods require the homogeneous Dirichlet boundary conditions in order to get the accurate order of convergence, see Ford et al.. [14]

\begin{tabular}{cccccccc}
\hline$\alpha$ & & $h=1 / 4$ & $h=1 / 8$ & $h=1 / 16$ & $\mathrm{~h}=1 / 32$ & $\mathrm{~h}=1 / 64$ & (theoretical orders) \\
\hline $5 / 8$ & FEM & $1.61 \mathrm{e}-2$ & $5.17 \mathrm{e}-3$ & $1.41 \mathrm{e}-3$ & $3.83 \mathrm{e}-4$ & $1.18 \mathrm{e}-4$ & \\
& & & 1.64 & 1.87 & 1.88 & 1.69 & $(0.25)$ \\
& G-L & $3.10 \mathrm{e}-1$ & $(1.60 \mathrm{e}-1)$ & $(8.15 \mathrm{e}-2)$ & $(4.11 \mathrm{e}-2)$ & $(2.05 \mathrm{e}-2)$ & \\
$3 / 4$ & & & 0.95 & 0.97 & 0.99 & 1.00 & \\
& & & & & \\
& GEM & $(1.29 \mathrm{e}-2$ & $3.15 \mathrm{e}-3$ & $8.90 \mathrm{e}-4$ & $2.57 \mathrm{e}-4$ & $8.18 \mathrm{e}-5$ & \\
& & 2.03 & 1.82 & 1.79 & 1.65 & $(0.50)$ \\
$7 / 8$ & FEM & $1.88 \mathrm{e}-1)$ & $(9.19 \mathrm{e}-2)$ & $(4.55 \mathrm{e}-2)$ & $(2.26 \mathrm{e}-2)$ & $(1.12 \mathrm{e}-2)$ & \\
& & 1.03 & 1.01 & 1.01 & 1.01 & \\
& G-L & $(1.19 \mathrm{e}-1)$ & $2.67 \mathrm{e}-3$ & $4.93 \mathrm{e}-4$ & $1.10 \mathrm{e}-4$ & $3.48 \mathrm{e}-5$ & \\
& & & $(4.54$ & 2.44 & 2.16 & 1.67 & $(0.75)$ \\
\hline
\end{tabular}

Table 3: The experimentally determined orders of convergence ("EOC") for the different $\alpha$ at $t_{N}=1$ in Example 5.3

The following two examples are related to the two-sided space-fractional derivatives.

Example 5.4. Consider the following linear elliptic space-fractional partial differential equation, with $1 / 2<$ $\alpha<1$,

$$
\begin{aligned}
& \mathcal{L}^{2 \alpha} u(x)=f(x), 0<x<1, \\
& u(0)=u(1)=0
\end{aligned}
$$

where $f(x) \in L^{2}(0,1)$. 
The purpose of this example is to find the experimentally determined order of regularity ("EOR") $r$ for the solution $u(x)$ of (72)-(73) with $f \in L^{2}(0,1)$, where we have $r=\alpha+\beta, 1 / 2<\alpha<1$ and

$$
\|u\|_{H_{0}^{r}(0,1)} \leq C\|f\| .
$$

Assume that this is true, following the proof of Lemma 3.1 we may show the error estimates

$$
\left\|u_{h}-u\right\| \leq C h^{2(r-\alpha)}
$$

where $u_{h}$ is the finite element approximation of $u$ and $h$ is the space step size. Choose the different space step sizes $h_{i}=1 / 2^{i}, i=2,3,4,5,6$. Let $e^{(i)}=\left\|u_{h}-u\right\|$ denote the $L^{2}$ norm obtained by using the space step sizes $h_{i}$. Since the exact solution is not available, we calculate the reference solution ( or 'true' solution) $u$ by using the very small space step size $h=2^{-10}$. Note that $e^{(i)} \leq C h_{i}^{2(r-\alpha)}$, which implies that the convergence order $2(r-\alpha)$ satisfies

$$
\log _{2}\left(e^{(i)} / e^{(i+1)}\right) \approx \log _{2}\left(h_{i} / h_{i+1}\right)^{2(r-\alpha)}=2(r-\alpha) .
$$

For the fixed $\alpha$, in Table 4, we calculate the experimentally determined orders of regularity with respect to the different space step sizes $h_{i}=1 / 2^{i}, i=2,3,4,5,6$ by using $r \approx \alpha+\frac{1}{2} \log _{2}\left(e_{n}^{(i)} / e_{n}^{(i+1)}\right)$.

In Table 4, we choose $f(x)=x^{1 / 4} \in H^{1 / 4-\epsilon} \subset L^{2}(0,1), \epsilon>0$ and provide the experimentally determined orders of regularity ("EOR") $r$ of $u$ and the theoretical orders of the regularity of $u$. For comparison purpose, we consider in Table 4 both the left-sided and two-sided space fractional derivative cases as expected. The theoretical regularity order in the left-sided space-fractional derivative case is proved to be $r \approx 2 \alpha-1+\beta, 0 \leq$ $\beta<1 / 2$, see Jin et al. [20] and to be $r \approx \alpha+\beta, 0<\beta<1 / 2$ for the two-sided space-fractional derivative case, see $[9,(5.4)]$. We observe that the experimentally determined order of regularity ("EOR") $r$ in the left-sided space-fractional derivative case is higher than the theoretical order. But it is not true for the

\begin{tabular}{|c|c|c|c|c|c|c|c|}
\hline$\alpha$ & & $h=1 / 4$ & $h=1 / 8$ & $h=1 / 16$ & $\mathrm{~h}=1 / 32$ & $\mathrm{~h}=1 / 64$ & ( theoretical $r$ ) \\
\hline \multirow[t]{4}{*}{$2 / 3$} & two-sided & $8.88 \mathrm{e}-2$ & $4.53 \mathrm{e}-2$ & $2.50 \mathrm{e}-2$ & $1.40 \mathrm{e}-2$ & $8.25 \mathrm{e}-3$ & \\
\hline & & & 1.15 & 1.09 & 1.08 & 1.05 & $(1.16)$ \\
\hline & Left-sided & $8.88 \mathrm{e}-2$ & $4.52 \mathrm{e}-2$ & $2.50 \mathrm{e}-2$ & $1.40 \mathrm{e}-2$ & $8.25 \mathrm{e}-3$ & \\
\hline & & & 1.15 & 1.09 & 1.09 & 1.05 & $(0.83)$ \\
\hline \multirow[t]{4}{*}{$3 / 4$} & two-sided & $2.82 \mathrm{e}-2$ & $1.34 \mathrm{e}-2$ & $6.56 \mathrm{e}-3$ & $3.24 \mathrm{e}-3$ & $1.62 \mathrm{e}-3$ & \\
\hline & & & 1.29 & 1.27 & 1.26 & 1.25 & $(1.25)$ \\
\hline & Left-sided & $2.82 \mathrm{e}-2$ & $1.34 \mathrm{e}-2$ & $6.56 \mathrm{e}-3$ & $3.23 \mathrm{e}-3$ & $1.62 \mathrm{e}-3$ & \\
\hline & & & 1.28 & 1.27 & 1.26 & 1.25 & $(1.00)$ \\
\hline \multirow[t]{4}{*}{$7 / 8$} & two-sided & $3.99 \mathrm{e}-3$ & $1.60 \mathrm{e}-3$ & $6.43 \mathrm{e}-4$ & $2.61 \mathrm{e}-4$ & $1.05 \mathrm{e}-4$ & \\
\hline & & & 1.53 & 1.53 & 1.52 & 1.53 & $(1.38)$ \\
\hline & Left-sided & $3.99 \mathrm{e}-3$ & $1.60 \mathrm{e}-3$ & $6.43 \mathrm{e}-4$ & $2.61 \mathrm{e}-4$ & $1.05 \mathrm{e}-4$ & \\
\hline & & & 1.53 & 1.53 & 1.52 & 1.53 & $(1.25)$ \\
\hline
\end{tabular}
two-sided space-fractional derivative case. Here the numbers in the brackets are the theoretical orders of regularity of $u$.

Table 4: The experimentally determined order of regularity ("EOR") for the different $\alpha$ in Example 5.4 in the Riesz spacefractional derivative and the left Riemann-Liouville fractional derivative cases, respectively

Example 5.5. , [9, Example 1] Consider the following linear space-fractional partial differential equation, with $1 / 2<\alpha<1$,

$$
\begin{aligned}
& \frac{\partial u(x, t)}{\partial t}+\mathcal{L}^{2 \alpha} u(x, t)=f(x, t), \quad 0<t<T, 0<x<1, \\
& u(0, t)=u(1, t)=0, \quad 0<t<T \\
& u(x, 0)=u_{0}(x), \quad 0<x<1
\end{aligned}
$$


where, with $C={ }_{2} F_{1}(\alpha, 1-\alpha ; 1+\alpha, x)$,

$$
f(x, t)=-e^{-t}\left(x-C x_{2}^{\alpha} F_{1}(\alpha, 1-\alpha ; 1+\alpha, x)\right)+e^{-t}\left(-\frac{1}{2} \frac{1}{\Gamma(2-2 \alpha)} x^{1-2 \alpha}+\frac{1}{2} \frac{1}{\Gamma(2-2 \alpha)}(1-x)^{1-2 \alpha}\right) .
$$

The exact solution is

$$
u(x, t)=e^{-t}\left(x-C x_{2}^{\alpha} F_{1}(\alpha, 1-\alpha ; 1+\alpha, x)\right),
$$

and the initial value $u_{0}(x)=x-C x^{\alpha}{ }_{2} F_{1}(\alpha, 1-\alpha ; 1+\alpha, x)$. Here the function ${ }_{2} F_{1}(\alpha, 1-\alpha ; 1+\alpha, x)$ is defined as in Ervin et al. [9, Definition 1].

By Theorems 3.8, we have, with some $0 \leq \beta<1 / 2,1 / 2<\alpha<1$,

$$
e_{N}^{(i)} \leq C h_{i}^{2 \beta}
$$

We use the same notations as in Example 5.2. In Table 5, we observe that for any $\alpha \in(1 / 2,1)$, the experimentally determined order of convergence ("EOC") is almost $2 \beta \approx 1$ with $\beta \approx 1 / 2$, which is consistent with the theoretical results in Theorem 3.8. It is also consistent with the numerical result in [9, Example 1] where the corresponding fractional elliptic problem is considered.

\begin{tabular}{cccccc}
\hline$\alpha$ & $h=1 / 4$ & $h=1 / 8$ & $h=1 / 16$ & $\mathrm{~h}=1 / 32$ & $\mathrm{~h}=1 / 64$ \\
\hline $5 / 8$ & $7.70 \mathrm{e}-2$ & $3.88 \mathrm{e}-2$ & $2.97 \mathrm{e}-2$ & $1.95 \mathrm{e}-2$ & $0.99 \mathrm{e}-2$ \\
& & 0.99 & 0.99 & 0.99 & 0.98 \\
$3 / 4$ & $1.99 \mathrm{e}-2$ & $9.95 \mathrm{e}-3$ & $4.97 \mathrm{e}-3$ & $2.43 \mathrm{e}-3$ & $1.22 \mathrm{e}-3$ \\
& & 1.01 & 1.01 & 1.01 & 1.00 \\
$7 / 8$ & $3.63 \mathrm{e}-3$ & $1.82 \mathrm{e}-3$ & $8.79 \mathrm{e}-4$ & $4.24 \mathrm{e}-4$ & $2.06 \mathrm{e}-4$ \\
& & 1.05 & 1.05 & 1.05 & 1.04 \\
\hline
\end{tabular}

Table 5: The experimentally determined orders of convergence ("EOC") for the different $\alpha$ at $t_{N}=1$ in Example 5.5

\section{Acknowledgements}

The authors are grateful to the Reviewers and the Associate Editor for their helpful comments.

\section{References}

[1] D. A. Benson, S. W. Wheatcraft, M. M. Meerschaert, Application of a fractional advection-dispersion equation, Water Resour.Res. 36 (2000) 1403-1412.

[2] W. Bu, Y. Tang, J. Yang, Galerkin finite element method for two-dimensional Riesz space fractional diffusion equations, J. Comput. Phys. 276 (2014) 26-38.

[3] A. Bueno-Orovio, D. Kay, K. Burrage, Fourier spectral methods for fractional-in-space reaction-diffusion equations, BIT Numer. Math. (54) 2014 937-954.

[4] K. Burrage, N. Hale, D. Kay, An efficient implicit FEM scheme for fractional-in-space reaction-diffusion equations, SIAM J. Sci. Comput. 34 (2012) A2145-A2172.

[5] L. A. Caffarelli, A. Vasseur, Drift diffusion equations with fractional diffusion and the quasi-geostrophic equation, Ann. of Math. 171 (2010) 1903-1930.

[6] X. Chen, F. Zeng, and G.E. Karniadakis, A tunable finite difference method for fractional differential equations with non-smooth solutions, Comput. Methods Appl. Mech. Engrg. 318 (2017) 193-214.

[7] W. Deng, J. S. Hesthaven, Discontinuous Galerkin methods for fractional diffusion equations, ESAIM:M2AN 47 (2013) 1845-1864.

[8] R. Du, Y. Yan, Z. Liang, A high-order scheme to approximate the Caputo fractional derivative and its application to solve the fractional diffusion wave equation, Journal of Computational Physics, in press, (2018).

[9] V. J. Ervin, N. Heuer, and J. P. Roop, Regularity of the solution to 1-d fractional order diffusion equations, Math. Comp. 87 (2018) 2273-2294.

[10] V. J. Ervin, J. P. Roop, Variational formulation for the stationary fractional advection dispersion equation, Numer. Methods Partial Differential Equations 22 (2006) 558-576. 
[11] W. Fan, F. Liu, X. Jiang, I. Turner, A novel unstructured mesh finite element method for solving the time-space fractional wave equation on a two-dimensional irregular convex domain, Fractional Calculus and Applied Analysis 20 (2017), $352-383$.

[12] L. Feng, F. Liu, I. Turner, Q. Yang and P. Zhuang, Unstructured mesh finite difference/finite element method for the 2d time-space Riesz fractional diffusion equation on irregular convex domains, Applied Mathematical Modelling 59 (2018), 441-463.

[13] G.J. Fix, J. P. Roop, Least squares finite-element solution of a fractional order two-point boundary value problem, Comput. Math. Appl. 48 (2004) 1017-1033.

[14] N. J. Ford, K. Pal, Y. Yan, An algorithm for the numerical solution of space-fractional partial differential equations, Computational Methods in Applied Mathematics 15 (2015) 497-514.

[15] N. J. Ford, M. Manuela Rodrigues, J. Xiao, Y. Yan, Numerical analysis of a two-parameter fractional telegraph equation, Journal of Computational and Applied Mathematics 249 (2013) 95-106.

[16] N. J. Ford, J. Xiao, and Y. Yan, Stability of a numerical method for a space-time-fractional telegraph equation, Computational Methods in Applied Mathematics 12 (2012) 1-16.

[17] Z. Hao and W. Cao, An improved algorithm based on finite differences for fractional boundary value problems with nonsmooth solution, Journal of Scientific Computing 73 (2017) 395-415.

[18] M. Ilic, F. liu, I. Turner, V. Anh, Numerical approximation of a fractional-in-space diffusion equation I, Frac. Calc. Appl. Anal. 8 (2005) 323-341.

[19] M. Ilic, F. liu, I. Turner, V. Anh, Numerical approximation of a fractional-in-space diffusion equation II: with nonhomogeneous boundary conditions, Frac. Calc. Appl. Anal. 9 (2006) 333-349.

[20] B. Jin, R. Lazarov, J. Pasciak, W. Rundell, Variational formulation of problems involving fractional order differential operators, Math. Comp. 84(2015) 2665-2700.

[21] B. Jin, R. Lazarov, J. Pasciak, Z. Zhou, Error analysis of finite element methods for space-fractional parabolic equations, SIAM J. Numer. Anal. 52 (2014) 2272-2294.

[22] C. Johnson, Numerical Solution of Partial Differential Equations by the Finite Element Method, Cambridge University Press, 1987.

[23] X. J. Li, C. J. Xu, A space-time spectral method for the time fractional diffusion equation, SIAM J. Numer. Anal. 47 (2009) 2108-2131.

[24] X. J. Li, C. J. Xu, Existence and uniqueness of the weak solution of the space-time fractional diffusion equation and a spectral method approximation, Commun. Comput. Phys. 8 (2010) 1016-1051.

[25] Z. Li, Z. Liang, Y. Yan, High-order numerical methods for solving time fractional partial differential equations, Journal of Scientific Computing, 71 (2017) 785-803.

[26] Y. Liu, Y. Yan, M. Khan, Discontinuous Galerkin time stepping method for solving linear space fractional partial differential equations, Applied Numerical Mathematics 115 (2017) 200-213.

[27] Z. Mao, G. E. Karniadakis, A spectral method (of exponential convergence) for singular solutions of the diffusion equation with general two-sided fractional derivative, SIAM J. Numer. Anal. 56 (2018) 26-49.

[28] M. M. Meerschaert, C. Tadjeran, Finite difference approximations for fractional advection-dispersion flow equations, J. Comput. Appl. Math. 172 (2004) 65-77.

[29] M. M. Meerschaert, H. Scheffler, C. Tadjeran, Finite difference methods for two-dimensional fractional dispersion equation, J. Comput. Phys. 211 (2006) 249-261.

[30] R. Metzler, J. Klafter, The random walk's guide to anomalous diffusion: a fractional dynamics approach, Phys. Rep. 339 (2000) 1-77.

[31] K. Pal, F. Liu, Y. Yan and G. Roberts, Finite difference method for two-sided space-fractional partial differential equations. In: I. Dimov, I. Farago and L. Vulkov (Eds.), Finite Difference Methods, Theory and Applications, 6th International Conference, (2014) 307-314.

[32] S. Picozzi, B. West, Fractional Langevin model of memory in financial markets, Phys. Rev. E 66 (2002), 046118

[33] I. Podlubny, Fractional Differential Equations, Academic Press, 1999.

[34] S. Qin, F. Liu, I. Turner, A two-dimensional multi-term time and space fractional Bloch-Torrey model based on bilinear rectangular finite elements, Communications in Nonlinear Science and Numerical Simulation 56 (2018), $270-286$.

[35] S. G. Samko, A. A. Kilbas, O.I. Marichev, Fractional Integrals and Derivatives: Theory and Applications (Translation from the Russian), Gordon and Breach, Amsterdam, 1993.

[36] R. K. Saxena, A. M. Mathai, H. J. Haubold, On generalized fractional kinetic equations, Physica A 344 (2004) $657-664$.

[37] C. Tadjeran, M. M. Meerschaert, H. Scheffler, A second-order accurate numerical approximation for the fractional diffusion equation, J. Comput. Phys. 213 (2006) 205-213.

[38] V. Thomée, Galerkin Finite Element Methods for Parabolic Problems, Springer-Verlag, Berlin, 2006.

[39] Q. Yang, F. Liu, I. Turner, Numerical methods for fractional partial differential equations with Riesz space-fractional derivatives, Appl. Math. Model. 34 (2010) 200-218.

[40] S. B. Yuste, L. Acedo, K. Lindenberg, Reaction front in an $A+B \rightarrow C$ reaction-subdiffusion process, Phys. Rev. E 69 (2004) 036126.

[41] S. B. Yuste, K. Lindenberg, Subdiffusion-limited $A+A$ reactions, Phys. Rev. Lett. 87 (2001) 118301.

[42] Y. Zhao, Y. Zhang, D. Shi, F. Liu, I. Turner, Superconvergence analysis of noncomforming finite element method for two-dimensional time fractional diffusion equations, Applied Mathematics Letters, 59 (2016) 38-47.

[43] Y. Zhao, Y. Zhang, F. Liu, I. Turner, Y. Tang, V. Anh, Analytical solution and nonconforming finite element approximation for the 2D multi-term fractional subdiffusion equation, Applied Mathematical Modelling, 40 (2016) 8810-8825.

[44] G. M. Zaslavsky, Topological Aspects of the Dynamics of Fluids Plasmas, Dordrecht, 1992.

[45] G.M. Zaslavsky, Chaos, fractional kinetics, and anomalous transport, Phys. Rep. 371 (2002) 461-580. 
[46] F. Zeng, F. Liu, C. Li, K. Burrage, I. Turner, and V. Anh, Crank-Nicolson ADI spectral method for the two-dimensional Riesz space-fractional nonlinear reaction-diffusion equation, SIAM J. Numer. Anal. 52 (2014) 2599-2622.

[47] F. Zeng, Z. Mao, G.E. Karniadakis, A generalized spectral collocation method with tunable accuracy for fractional differential equations with end-point singularities, SIAM J. Sci. Comput. 37 (2017) A2710A2732.

[48] H. Zhang, F. Liu and V. Anh, Galerkin finite element approximations of symmetric space-fractional partial differential equations, Appl. Math. Comput. 217 (2010) 2534-2545. 